\title{
Antimicrobial Activities of Novel 3-Substituted $[1,2,4]$ Triazolo[4,3-b]pyridazines Derivatives
}

\author{
Jayaraman Sembian Ruso, Nagappan Rajendiran*, Chowdappa Srinivas ${ }^{\dagger}$, Konappa Narasimha murthy ${ }^{\dagger}$, \\ and Krishnamurthy Soumya ${ }^{\dagger}$ \\ Department of Polymer Science, Maramalai Campus, University of Madras, Guindy, Chennai-600025, India. \\ *E-mail: nrajendiar@yahoo.com \\ ${ }^{\dagger}$ Department of Microbiology, Janabharathi Campus, Bangalore-560056, India
}

(Received February 26, 2014; Accepted June 12, 2014)

\begin{abstract}
A noval derivatives of 3-substituted[1,2,4]triazolo[4,3-b]pyridazine (4a-4o) and 7,8,9,10-tetrahydrobenzo[4,5] thieno[2,3- $d][1,2,4]$ triazolo[4,3-b]pyridazine $(\mathbf{7 a}-7 \mathbf{l})$ is prepared by the reaction of heteroaryl hydrazone from the aldehyde and pyridazinohydrazine derivative, followed by subjecting the intermediate directly to oxidative cyclization employing the mixture of $\mathrm{Me}_{4} \mathrm{NBr}$ and Oxone. These derivatives were subjected to preliminary antimicrobial activities against microorganism. All these compounds exhibit good to moderate activity.
\end{abstract}

Key words: 1,2,4 Triazolopyridazine, Aromatic aldehyde antifungal and antibacterial

\section{INTRODUCTION}

Omnipresent interest on the synthesis of 1,2,4 triazole derivatives are owing to their diverse use in organocatalysis, materials science and biological applications. Most of the 1,2,4-triazole structural motif exhibit a wide spectrum of biological properties including antimicrobial, antitubercular, serotonergic, anti-allergy, CNS depressant, antiinflammatory, anticonvulsants and anti-cancer activities. ${ }^{1}$ The recent exploratory result reveals that 1,2,4 triazoles derivatives performed as inhibitor of HIV-integrase and methionine aminopeptidase. ${ }^{2}$ Besides, many powerful and popular drugs constituted with 1,2,4 triazoles system are commercially available in the market e.g., Fluconazole, Itraconazole, varozole, voriconazole, Loreclezole, Anastrolzole and triazolam (Figure 1).

Attention to synthesis of several biologically active compounds with pyridazine ring is an emerging practice due to its numerous activity against microbial, fungal, viral, tumor, hypertensive, tubercular and cancer cells. ${ }^{3}$ In addition, a heterocyclic system extended with the pyridazines moiety are found to be an important pharmacore to construct a potentially new bio-active molecule in modern medicinal chemistry. ${ }^{4}$ In particularly, the physiological and pharmacological activity can be attractively enhanced by introducing a thieno group in to the pyridazine motif. ${ }^{5}$ Furthermore, triazolopyridazine derivatives have been reported to possess anti-HAV activity, c-Met kinase inhib- itor, $\mathrm{GABA}_{\mathrm{A}}$ and adenine receptors. ${ }^{6}$

Most common and widely used method to construct 1,2,4-triazole system is the oxidative cyclization of hydrazone. Very recently, we have reported the one pot synthesis of triazolopyridazine derivative and thieno-triazolopyridazine derivatives using $\mathrm{Me}_{4} \mathrm{NBr}$ and oxone. ${ }^{7}$ As a continuation, our interest to investigate the antimicrobial activities of the noval triazolopyridazine derivatives, we report herein the antibacterial and antifungal activity of the compound 3 -substituted $[1,2,4]$ triazolo[4,3-b]pyridazine (4a-4o) and 7,8,9,10-tetrahydrobenzo[4,5] thieno[2,3- $d][1,2,4]$ triazolo $[4,3-b]$ pyridazine $(\mathbf{7 a - 7 1})$ against the bacterial strain (Staphylococcus aureus and Bacillus subtilis-Gram positive, Escherichia coli and Pseudomonas aeruginosa-Gram negative) and fungi (Aspergillus niger and Aspergillus flavus) using the broth dilution method.

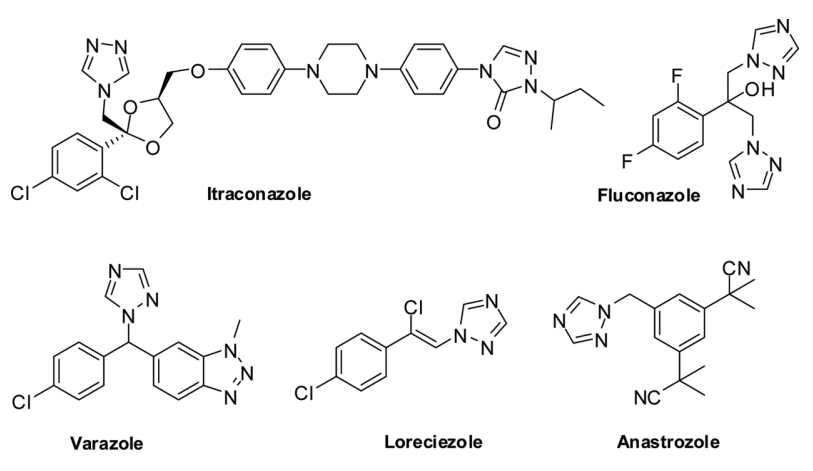

Figure 1. Drugs containing 1,2,4 triazole moiety. 


\section{EXPERIMENTAL}

All reagents were purchased from commercial suppliers and were used without purification. Melting points were determined in Buchi B-545melting point apparatus and were uncorrected. ${ }^{1} \mathrm{H}$ NMR and ${ }^{13} \mathrm{C}$ NMR spectra were recorded on a Bruker Advance-400 \& 300 NMR MHZ spectrometer in DMSO- $d_{6} \& \mathrm{CDCl}_{3}$ solution using TMS as an internal reference and ${ }^{13} \mathrm{C}$ NMR were recorded at 100 \& $75 \mathrm{MHz}$. Mass spectra were recorded on LC-MS-Agilent 1200 series, Carbon, Hydrogen, Nitrogen and Sulphur were analyzed on Elementor instrument. All these compounds were purified by flash column Chromatography using 230400 mesh silica gel.

\section{General Procedure for the Synthesis of 3-Substituted Triazolopyridazine}

A mixture of corresponding hydrazinylpyridazine 1 or 5 $(1 \mathrm{mmol})$ and aldehyde $2(1.1 \mathrm{mmol})$ in ethanol $(5 \mathrm{~mL})$ was heated at $60^{\circ} \mathrm{C}$ for $0.5 \mathrm{~h}$. The formation of hydrazone was checked by TLC and the reaction mixture was cooled to rt. Oxone $(1.5 \mathrm{mmol})$ was added to the mixture at rt followed by tetramethyl ammonium bromide $(0.2 \mathrm{mmol})$ and the resulting mixture was heated at $60^{\circ} \mathrm{C}$ for another $5 \mathrm{~h}$. The mixture was cooled to rt and extracted with dichloromethane $(2 \times 25 \mathrm{~mL})$, dried over anhydrous sodium sulfate and concentrated to obtain a residue which was purified by column chromatography using hexane/ethyl acetate as eluent to furnish the desired triazolopyridazines $\mathbf{4}$ and $\mathbf{7}$ (See reference no; 7 for supporting information).

\section{RESULTS AND DISCUSSION}

Target compounds $\mathbf{4 a}-\mathbf{4 o}$ and $\mathbf{7 a}-\mathbf{7 l}$ were prepared by one-pot synthesis. At first, hydrazinylpyridazine ( 1 or $\mathbf{5})$ was treated with various aldehydes to obtain the heteroarylhydrazones ( $\mathbf{3}$ or $\mathbf{6}$ ) which was subjected directly to oxidative cyclization with the mixture of $\mathrm{Me}_{4} \mathrm{NBr}(20 \mathrm{~mol} \%)$ and oxone (1.5 equiv.) to acquire the triazolopyridazine derivatives of $\mathbf{4 a - 4 0}$ and $7 \mathbf{a}-\mathbf{7 1}$ (Scheme $1 \& 2$ ) in $80-95 \%$ yields. ${ }^{8}$

Twenty seven compounds were synthesised and their structure was confirmed by NMR, mass spectroscopy and elemental analysis. All the synthesized compounds $\mathbf{4 a}-\mathbf{4 o}$ and $7 \mathbf{a}-7 \mathbf{l}$ were screened for their in vitro antibacterial activity against Gram-positive (Staphylococcus aureus and Bacillus subtiis) and Gram-negative (Escherichia coli and Pseudomonas aeruginosa) bacteria strains using broth dilution method for determination of MIC. ${ }^{9}$ This activity was compared to a well-known commercial antibiotic, such as

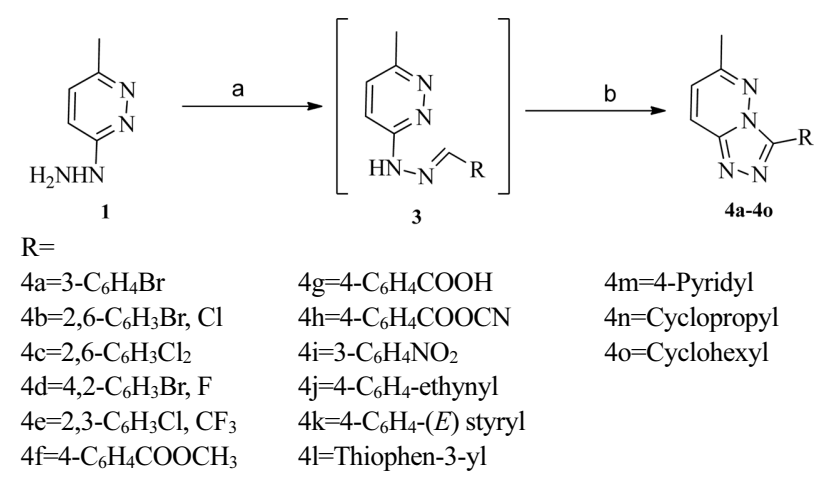

Scheme 1. (a) Aldehyde, ethanol, $60{ }^{\circ} \mathrm{C}, 30 \mathrm{~min}$ (b) teramethylammonium bromide/oxone, $60^{\circ} \mathrm{C}, 5 \mathrm{~h}$.

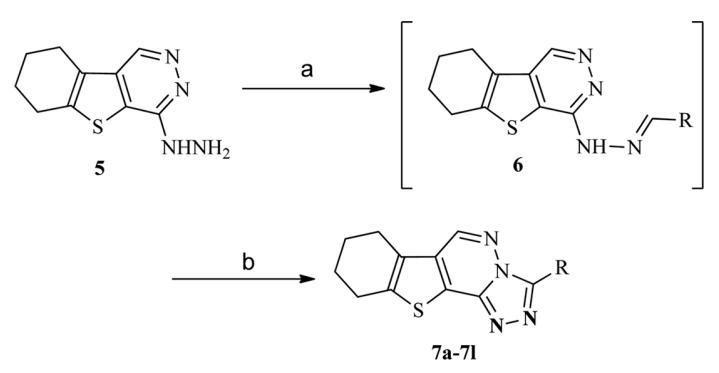

$\mathrm{R}=$

$7 \mathrm{a}=3-\mathrm{C}_{6} \mathrm{H}_{4} \mathrm{Br} \quad 7 \mathrm{~h}=4-\mathrm{C}_{6} \mathrm{H}_{4} \mathrm{COOMe}$

$7 \mathrm{~b}=4-\mathrm{C}_{6} \mathrm{H}_{3} \mathrm{Br} \quad$ 7i=4- $\mathrm{C}_{6} \mathrm{H}_{4}$-Allyloxy

$7 \mathrm{c}=2-\mathrm{C}_{6} \mathrm{H}_{4} \mathrm{CF} \quad 7 \mathrm{j}=4-\mathrm{C}_{6} \mathrm{H}_{4}-(E)$ Styryl

$7 \mathrm{~d}=2,6-\mathrm{C}_{6} \mathrm{H}_{3} \mathrm{Cl}_{2} \quad 7 \mathrm{k}=$ Cyclopropyl

$7 \mathrm{e}=2,4-\mathrm{C}_{6} \mathrm{H}_{3} \mathrm{OMe}, \mathrm{Br} \quad 7 \mathrm{l}=n$-hexyl

$7 \mathrm{f}=2,4,6-\mathrm{C}_{6} \mathrm{H}_{2}(\mathrm{OMe})_{3}$

$7 \mathrm{~g}=4-\mathrm{C}_{6} \mathrm{H}_{4} \mathrm{OPh}(4 \mathrm{~F})$

Scheme 2. (a) Aldehyde, ethanol, $60^{\circ} \mathrm{C}, 30 \mathrm{~min}$ (b) teramethylammonium bromide/oxone, $60{ }^{\circ} \mathrm{C}, 5 \mathrm{~h}$.

ampicillin. Screening results revealed that the compounds 4a-4o and 7a-7l displayed good to moderate activities towards the tested antibacterial strains. On the basis of the zone of inhibition produced against test bacteria, compounds 4d, 4I, 4m, 7f and $7 \mathbf{i}$ found to have significant activity against both Gram-positive and Gram-negative bacterial strains, whereas the compounds $\mathbf{4 c}, \mathbf{4 g}$ and $\mathbf{7 c}$ showed good activity against Gram-positive bacteria, and also $4 \mathbf{i}$ and $7 \mathrm{~g}$ exhibited potent activities against the Gram-negative bacterial strains. These results are summarised in (Table 1, Figure 2).

Rest of all compounds displayed week activity against the tested bacterial strains. In these case, the increased activity could be attributed the presence of fluro, chloro, acid, ester, nitro, pyridyl, thiophene, allyl and phenoxy group in these compounds. All the twenty seven compounds were also subjected to their antifungal activities against the fungi namely, Aspergillus flavus and Aspergillus Niger. 
Table 1. In vitro antibacterial screening results of the tested compounds $\mathbf{4 a - 4 0}$ and $\mathbf{7 a}-\mathbf{7 l}$

\begin{tabular}{|c|c|c|c|c|}
\hline \multirow{2}{*}{ Compounds } & \multicolumn{4}{|c|}{ Inhibition zone diameter (mm/mg sample) } \\
\hline & S. aureus & B. subtiis & E. coli & $P$. aeruginosa \\
\hline $4 \mathbf{4 a}$ & 6 & 6 & 8 & 6 \\
\hline $4 b$ & 7 & 6 & 7 & 7 \\
\hline $4 c$ & 10.4 & 10 & 9 & 8 \\
\hline 4d & 11 & 12 & 12 & 11 \\
\hline $4 e$ & 8 & 9 & 7 & 8 \\
\hline 4f & 9 & 8 & 9 & 9 \\
\hline $4 g$ & 10.3 & 12 & 8 & 7 \\
\hline $4 h$ & 6 & 9 & 7 & 9 \\
\hline $4 \mathbf{i}$ & 8.6 & 9.5 & 10.5 & 10 \\
\hline $4 j$ & 8 & 9 & 6 & 7 \\
\hline $4 k$ & 6 & 8 & 5 & 4 \\
\hline 41 & 10 & 11 & 10 & 11 \\
\hline $4 m$ & 11.0 & 12 & 11 & 10.7 \\
\hline $4 n$ & 8 & 7 & 6 & 5 \\
\hline 40 & 6 & 7 & 5 & 6 \\
\hline $7 a$ & 8 & 7 & 8 & 6 \\
\hline $7 b$ & 7 & 6 & 7 & 7 \\
\hline $7 c$ & 11 & 12 & 8 & 7 \\
\hline $7 d$ & 7 & 8 & 7 & 9 \\
\hline $7 e$ & 8 & 9 & 8 & 7 \\
\hline $7 f$ & 10.2 & 11 & 11 & 12 \\
\hline $7 \mathrm{~g}$ & 9 & 9 & 10.5 & 10.2 \\
\hline $7 \mathrm{~h}$ & 8 & 9 & 7 & 9 \\
\hline $7 \mathbf{i}$ & 10.5 & 10 & 10 & 11.3 \\
\hline $7 \mathbf{j}$ & 8 & 7 & 8 & 6 \\
\hline $7 k$ & 6 & 7 & 5 & 4 \\
\hline 71 & 7 & 8 & 5 & 6 \\
\hline Amphicilin & 12 & 14 & 14 & 12 \\
\hline
\end{tabular}

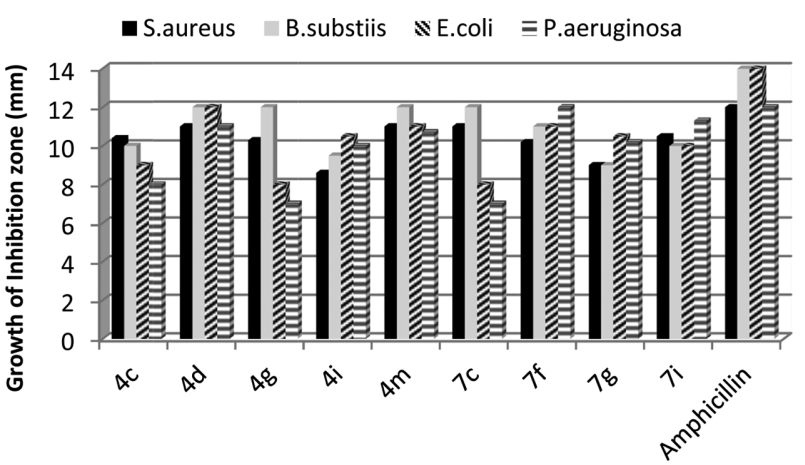

Figure 2. Diameter of growth of inhibition of selected compounds of antibacterial activity.

Fluconazole was used as standard for the comparison of antifungal activity of the compounds $4 \mathbf{a}-\mathbf{4 0}$ and $7 \mathbf{a}-\mathbf{a} 7 \mathbf{l}$.

This result indicated that almost all the compounds have week to good antifungal activity against the test fungi. The
Table 2. In vitro antifungal screening results of the tested compounds $4 \mathbf{a} 40$ and $7 \mathbf{a}-7 \mathbf{l}$.

\begin{tabular}{|c|c|c|}
\hline \multirow{2}{*}{ Compounds } & \multicolumn{2}{|c|}{ Mycelial growth of inhibition (\%) } \\
\hline & Aspergillus flavus & Aspergillus niger \\
\hline $4 \mathbf{a}$ & 40.2 & 52.7 \\
\hline $4 b$ & 44.5 & 45.3 \\
\hline $4 c$ & 52.4 & 43.2 \\
\hline $4 d$ & 61.1 & 62.3 \\
\hline $4 e$ & 43.2 & 44.3 \\
\hline $4 f$ & 42.3 & 43.5 \\
\hline $4 g$ & 61.8 & 60.4 \\
\hline $4 h$ & 61.2 & 61.0 \\
\hline $4 \mathbf{i}$ & 45.2 & 44.7 \\
\hline $4 j$ & 43.6 & 43.8 \\
\hline $4 \mathbf{k}$ & 41.2 & 2.8 \\
\hline 41 & 54.5 & 53.7 \\
\hline $4 m$ & 43.4 & 44.3 \\
\hline $4 n$ & 53.8 & 52.5 \\
\hline 40 & 43.4 & 40.6 \\
\hline $7 \mathbf{a}$ & 45.7 & 47.4 \\
\hline $7 \mathbf{b}$ & 45.4 & 43.8 \\
\hline $7 \mathrm{c}$ & 61.5 & 59.8 \\
\hline $7 d$ & 40.2 & 56.4 \\
\hline $7 e$ & 47.4 & 49.5 \\
\hline $7 f$ & 61.8 & 62.3 \\
\hline $7 \mathrm{~g}$ & 44.2 & 48.7 \\
\hline $7 \mathrm{~h}$ & 45.2 & 49.6 \\
\hline $7 \mathbf{i}$ & 52.7 & 56.5 \\
\hline $7 \mathbf{j}$ & 44.7 & 48.9 \\
\hline $7 k$ & 40.1 & 59.4 \\
\hline 71 & 43.9 & 58.4 \\
\hline Flucanozole & 72 & 80 \\
\hline
\end{tabular}

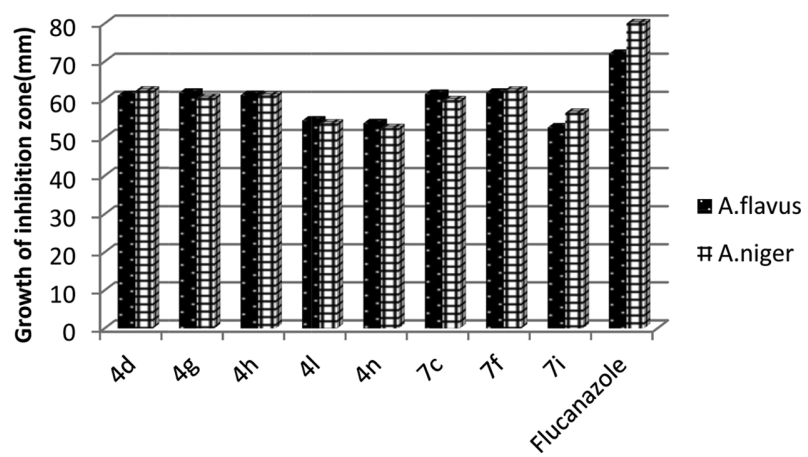

Figure 3. Diameter of growth of inhibition of selected compounds of antifungal activity.

compound 4d, $\mathbf{4 g}, \mathbf{4 h}, \mathbf{7 c}$, and $7 \mathbf{f}$ were found to have maximum inhibition zone $(77 \%)$ whereas the compound $\mathbf{4 l}, \mathbf{4 n}$ and $7 \mathbf{i}$ exhibited more than $65 \%$ inhibition zone against fungi, Aspergillus flavus and Aspergillus niger (Table 2, Figure 3). 
In conclusion, we have synthesized a novel series 3-methylhydrazinopyridazine (4a-4o) and 4-hydrazinyl-6,7,8,9tetrahydro[1] benzothieno[2,3- $d$ ]pyridazine derivative (7a-7l) using the mixture of $\mathrm{Me}_{4} \mathrm{NBr}$ and Oxone. All newly synthesized compounds were tested for their antimicrobial activities and showed fairly to good activities against the bacteria and fungi. However, in whole series, compound 4d and $\mathbf{4 f}$ possess potent antimicrobial activity.

Acknowledgments. Publication cost of this paper was supported by the Korean Chemical Society.

\section{REFERENCES}

1. (a) Ulusoy, N.; Gursoy, A.; Otuk, G. II Farmaco2001, 56, 947-952. (b) Papakostantinou, S.; Garoufalias, N.; Pouli, P.; Marakos, A.; Ladas, A.C. II Farmaco 2002, 57, 973977. (c) Kucukguzel, I. I.; Kucukguzel, S. G.; Rollas, S.; Kiraz, M. Bioorg. Med. Chem. Lett. 2001, 11, 17031707. (d) Patel, N. B.; Khan, I. H.; Rajani, S. D. Eur. J. Med. Chem. 2010, 45, 4293-4299. (e) Shiradkar, M.; Kumar, G. V. S.; Dasari, V.; Tatikonda, S.; Akula, K. C.; Shah, R. Eur. J. Med. Chem. 2007, 42, 807-816. (f) Parmar, S. S.; Gupta, G. A. K.; Singh, H. H.; Gupta, T. K. J. Med. Chem. 1972, 15, 999-1000. (g) Wang, L. C.; Tu, C. H.; Wang, J. H.; Lee, G. H. Molecules 2006, 11, 169-176. (h) Zitouni, G. T.; Kaplanciki, Z. A.; Ozdemir, A.; Chevallet, P.; Kandilci, H. B.; Gumusel, B. Arch. Pharm. Chem. Life. Sci. 2007, 11, 586-590. (i) Sarva, M. C.; Romeo, G.; Guerrera, F.; Siracusa, M.; Alerno, L.; Russo, F.; Cagnotto, A.; Goegan, M.; Mennin, T. Cheminform. 2010, 33, 2002-2004. (j) Holla, B. S.; Veerendra, B.; Poojary, M. K. Eur. J. Med. Chem. 2003, 38, 759-767. (k) Parmar, S. S.; Chaudhary, M.; Chaudhary, S. K.; Kumar, S.; Spiro, H. R. J. Pharm. Sci. 1977, 66, 971-975.

2. (a) Shimizu, S.; Endo, T.; Izumi, K.; Mikamiyama, H. Org. Process Res. Dev. 2007, 11, 1055-1058. (b) Marino, J. P., Jr.; et al. J. Med. Chem. 2007, 50, 3777-3785.

3. (a) Unqureanu, M.; Mangalagiu, I.; Grosu, G.; Petrovanu, M. Ann. Pharm. Fr. 1997, 55, 69-72. (b) Galtie, C.; Mavel, S.; Snoeck, R.; Andre, G.; Pannecouque, C.; Witvrouw, M.; Balzarini, J.; Clercq, E. D.; Gueiffier, A. Antivir. Chem. \& Chemother 2003, 14, 177-182. (c) Islam, M.; Siddiqui,
A. A.; Rajesh, R. Acta Pol Pharm. 2008, 65, 441-447. (d) Yassin, F. A. Chem. Heterocycl. Comp. 2009, 45, 997-1003. (e) Zhou, J.; Fan, H. T.; Song, B. A.; Jin, L. H.; Bhadury, P. S.; Hu, D. Y.; Yang, S. Phosphorus, Sulfur, Silicon Relat. Elem. 2010, 186, 81-87. (f) Bloomer, L. C.; Wotring, L. L.; Townsend, L. B. Cancer Res. 1982, 42, 100-106. (g) Katz, D. J.; Wise, D. S.; Townsend, L. B. J. Med. Chem. 1982, 25, 813-321. (h) Corina, M.; Casiraghi, G.; Zetta, L. C. J. Org. Chem. 1991, 56, 5466-5468. (i) Abubshait, S. A. Molecules 2007, 12, 25-42. (j) Yokoyama, M.; Tanabe, T.; Toyoshima, A.; Togo, H. Chem Lett. 1994, 517, 265-268. (k) Mantu, D.; Luca, M. C.; Moldoveanu, C.; Zbancioc, G.; Mangalagiu, I. Eur. J. Med. Chem. 2010, 45, 51645168. (l) Malinka, W.; Redizicka, A.; Lozach, O. II Farmaco 2004, 59, 457-462.

4. (a) Albright, J. D.; Moran, D. P.; Wright, W. B.; Collins, J. P.; Beer, B.; Lipa, A. S.; Greenblatt, E. N. J. Med. Chem. 1981, 24, 592-600. (b) Biagi, G.; Giorgi, I.; Livi, O.; Manera, C.; Scartoni, V.; Lucacchini, A.; Senatore, G. II Farmaco 1996, 51, 601-608. (c) Atack, J. R.; et al. J. Pharmacol. Exp. Ther. 2010, 332, 17-25. (d) Licata, S. C.; Platt, D. M.; Rüedi-Bettschen, D. R.; Atack, J. R.; Dawson, G. R.; Van Linn, M. L.; Cook, J. M.; Rowlett, J. K. Neuropharmacology. 2010, 58, 357-364.

5. (a) El-Dean, A. M. K.; Gaber, A. E. M.; El-Gaby, M. S. A.; Eyada, H. A. Cheminform. 2004, 35, 321-344. (b) Singh, A. K.; Hedge, G. L.; Khanum, S. A.; Shashikanth, S. Indian J. Pharm. Sci. 2005, 67, 210-215. (c) Pieretti, S.; Ominici, L. D.; Di Giannuario, A.; Cesari, N.; Dal Piaz, V. Life Sci. 2006, 79, 791-800. (d) Zaher, A. F. A.; Refaat, H. M.; Fawzy, O. M. Bull. Fac. Pharm. Cairo. Univ. 2009, 47, 69-75.

6. (a) Street, L. J.; et al. J. Med. Chem. 2004, 47, 36423657. (b) Shamroukh, A. H.; Ali, M. A. Arch. Pharm. 2008, 341, 223-230. (c) Albrecht, B. K.; et al. J. Med. Chem. 2008, 51, 2879-2882.

7. Sembian Ruso, J.; Rajendiran, N.; Senthilkumaran, R. J. Korean Chem. Soc. 2013, 57(5), 606. http://dx.doi.org/ 10.5012/jkcs.2013.57.5.606.

8. National committee for Clinical Laboratory Standards, Methods for Dilution Antimicrobial Susceptibility Test for Bcateria that Grow Aerobically, Approved Standard-Fith Edition. M7-A5 Ed. NCCLS, Wayne, PA, 2000, 1-33.

9. Thieno-Pyridazine derivative $\mathbf{5}$ was synthesized from 3formyl-4,5,6,7-tetrahydrobenzo[ $b]$ thiophene-2-carboxylic acid in four steps. 